\title{
A search for radio counterparts to Chandra ULX candidates
}

\author{
D. Pérez-Ramírez ${ }^{1, \star}$, M. Mezcua $^{2}$, S. Leon ${ }^{3}$, and M.D. Caballero-García ${ }^{4}$ \\ 1 Universidad de Jaén, Campus Las Lagunillas, s/n, E-23070, Jaén, Spain \\ 2 Max Planck Institute for Radio Astronomy, Auf dem Hügel 69, 53121 Bonn, Germany \\ 3 Joint Alma Observatory/ESO, Las Condes, Santiago, Chile \\ ${ }^{4}$ University of Cambridge, Institute of Astronomy, Cambridge CB3 OHA, UK
}

The dates of receipt and acceptance should be inserted later

Key words X-rays: binaries, radio continuum: galaxies, catalogs, surveys

\begin{abstract}
We present a systematic search for radio counterparts to Ultra Luminous X-ray (ULX) source candidates based on a crosscorrelation of the Swartz et al. (2004) ULX catalogue based on Chandra data and the FIRST radio survey. We find seven cases of conspiscuous peaks of radio emission that could be associated to ULX sources. Among these seven ULX radio candidates, three X-ray sources are located within 5" of the FIRST radio peaks. These three cases are shown and discussed individually.
\end{abstract}

(c) 2010 WILEY-VCH Verlag GmbH \& Co. KGaA, Weinheim

\section{Introduction}

The nature of Ultraluminous X-ray Sources (ULXs) is still under debate. Different models have been proposed to explain the possible physical mechanisms at work that give rise to the high X-ray luminosities observed (e.g. extragalactic microquasars, King et al. 2001; stellar mass black holes with super Eddington radiation, Begelman 2002; background AGN along the line of sight, Irwin, Bregman \& Athey 2004; intermediate mass black holes, Colbert \& Mushotzky 1999; or a consequence of a new ultraluminous accretion state, i.e. a transition between Eddington and super-Eddington accretion flows, Gladstone, Roberts \& Done 2009).

New X-ray surveys and associated ULX catalogues now available have compiled lists of potential ULX candidates. These catalogues are based on data from specific missions extracted from their corresponding archives. Earlier catalogues were based on ROSAT HRI data, as the one by Colbert \& Ptak (2002) that registered a total of 54 galaxies with 87 possible associated ULX candidates. These numbers increased with the catalogue by Liu \& Mirabel (2005), who compiled individual cases from published literature until April 2004 resulting in a total of 85 galaxies and 229 ULX new cases. Liu \& Bregman (2005) presented a more complete survey based also on ROSAT HRI data, where the number of recorded galaxies in the catalogue reached 313 , and their possible associated ULXs increased to 562. In a previous work (see Sánchez-Sutil et al. 2006) we initiated a systematic search of ULX radio counterparts mainly based on the cross-identification of the Liu \& Bregman (2005) catalogue and the FIRST catalogue, resulting in a total of 11 previously unreported matches, with a estimated positional uncertainty of $\sim 10^{\prime \prime}$.

\footnotetext{
* D. Pérez-Ramírez: e-mail: dperez@ujaen.es
}

The most promising data at the present time come from the Chandra mission due to its high spatial resolution and improved astrometry. In this sense, the catalogue by Swartz et al. (2004), hereafter Sw04, based on Chandra data is the only compiled list of potential ULXs with an estimated positional uncertainty of about $1-2^{\prime \prime}$.

The study of ULX radio counterparts can give us a clue to establish the ULX morphology, spectral index and variability properties. However, a radio counterpart for an ULX has only been found in a very few cases (Kaaret et al. 2003; Körding et al. 2005; Miller et al. 2005; Sánchez-Sutil et al. 2006).

We continue searching for possible radio counterparts to ULX candidates, now taking advantage of the published Chandra data catalogue. The work has been done following a similar approach to the one cited in Mushotzky (2004). The main results of our work reveal several new positional coincidences of FIRST radio sources with potential ULXs. In this paper we include both radio maps and individual discussion of the coincidence cases of special interest.

\section{Cross-identification and procedure}

We cross-identified the Sw04 catalogue, based on Chandra data, and the FIRST radio catalogue. Sw04 compiled a sample of 154 ULX candidates identified in 82 galaxies. These galaxies belong to different Hubble morphological types and also present distinct characteristics which make the sample more complete. The catalogue sample was also cleared for ULXs with positions located at less than $5^{\prime \prime}$ from the nucleus.

We used the catalogue of the VLA FIRST survey (Becker et al. 1995) that was released on April 2003 and was derived from the 1993 through 2002 observations (White et 
Table 1 Selected ULX sources with possible radio counterparts

\begin{tabular}{ccccccccc}
\hline \hline NGC & $\begin{array}{c}\text { ULX } \\
\text { Name }\end{array}$ & $\begin{array}{c}\alpha_{\text {J2000.0 }} \\
(\text { FIRST) }\end{array}$ & $\begin{array}{c}\delta_{\text {J2000.0 }} \\
(\text { FIRST) }\end{array}$ & $\begin{array}{c}\text { Separation } \\
\text { from nucleus } \\
\left({ }^{\prime}\right)\end{array}$ & $\begin{array}{c}\text { Radio vs. } \\
\text { X-ray offset } \\
\left({ }^{\prime \prime}\right)\end{array}$ & $\begin{array}{c}\text { Peak } \\
\text { Flux Density } \\
(\mathrm{mJ} \text { y/beam) }\end{array}$ & $\begin{array}{c}\text { Integrated } \\
\text { Flux } \\
(\mathrm{mJy})\end{array}$ & $\begin{array}{c}\text { RMS } \\
(\mathrm{mJy} / \mathrm{beam})\end{array}$ \\
\hline 4490 & $\mathrm{X} 1$ & $12^{\mathrm{h}} 30^{\mathrm{m}} 29^{\mathrm{s}} .46$ & $+41^{\circ} 39^{\prime} 27.4^{\prime \prime}$ & 1.5 & 1.1 & 3.34 & 7.17 & 0.156 \\
5194 & $\mathrm{X} 2$ & $13^{\mathrm{h}} 29^{\mathrm{m}} 50^{\mathrm{s}} .65$ & $+47^{\circ} 11^{\prime} 54.6^{\prime \prime}$ & 0.4 & 0.4 & 1.20 & 3.20 & 0.139 \\
5775 & $\mathrm{X} 4$ & $14^{\mathrm{h}} 54^{\mathrm{m}} 00^{\mathrm{s}} .98$ & $+03^{\circ} 31^{\prime} 29.0^{\prime \prime}$ & 1.4 & 4.1 & 2.94 & 4.04 & 0.139 \\
\hline
\end{tabular}

al. 1997). This survey presents a good sensitivity ( $\mathrm{rms} \sim$ $0.15 \mathrm{mJy})$ and angular resolution $\left(\sim 5^{\prime \prime}\right)$.

We based the cross-identification on the positional coincidence of the FIRST and the Chandra ULX entries within less than a certain error which depends mainly on the relative accuracy of the instruments involved. We established a radius of $5^{\prime \prime}$, which is roughly the maximum offset typically expected from the combined FIRST-Chandra astrometric errors. Not all the Sw04 sample galaxies were observed by the FIRST survey. We found FIRST radio data for 37 out of the 82 galaxies from the Sw04 sample, so only $43 \%$ of the Sw04 ULX parent galaxies were present.

From these 37 FIRST galaxies, half of them presented their ULX candidates located in regions where no radio emission was detected in the radio maps. The other half of the galaxy sample, this is 8 out of 19 , presented very poor signal to noise ratio radio maps. It was thus not possible to check radio coincidences for these cases.

Although the Sw04 catalogue does not contain any candidate within 5" of the nucleus, in 4 out of the 11 galaxies left, the matched radio source lies closer to the nucleus than the ULX candidate which might imply that the source could be nuclear in origin, and therefore, not a true ULX.

We ended up with 7 cases. We visually checked the matchesar and looked for compact radio counterpart candidates, which are the expected appearance of ULXs at the limited FIRST angular resolution and are compatible with the most plausible physical scenarios. However, extended radio sources were also considered if they were located within knotty optical features or spiral arms as possibly related to HII regions, since HII regions represent another suggested ULX scenario (Miller et al. 2005). Among these matches we report in the current work three cases for which the offset between the $\mathrm{X}$-ray source and the peak of compact radio emission is less than 5", and are located at distances larger than 20" from the nucleus within the galaxy disk. We present details for these cases in Table 1, where the FIRST estimated coordinates, the separation of the ULX with respect to the nucleus of the galaxy as given in NED, the radio/X-ray offset, peak flux density, integrated flux density, and rms are given respectively from Columns 3 to 9 . The numeration used to coin the ULXs responds to the order of these sources as they appear in the Sw04 list.

We looked for possible counterparts in the SIMBAD database for each of the selected sources, finding only a previous citation by Roberts et al. (2002) for one of the ULX candidates in the galaxy NGC 4490, who suggested that the source could be a supernova remnant (SNR). Figures 1 to 3 show the optical and radio fields to illustrate their positional coincidence. The optical images were retrieved from the Digitized Sky Survey (DSS) (Lasker et al. 1990), while the radio ones were taken from FIRST.

\section{Comments on selected cases}

This section is devoted to provide a more detailed individual discussion on the most interesting cases found in the crossidentification process.

\subsection{ULX 1 in NGC 4490}

NGC 4490 is a galaxy classified as SBm, a system close to be edge-on. It presents a knotty center but does not exhibit apparent nucleus or bulge.

Sw04 listed 5 potential ULXs associated to this galaxy. After close inspection we found that 4 of them are within the galaxy disk. The FIRST radio emission is limited to a smaller region close to the nucleus rather than covering the whole disk. We found a clear coincidence of ULX 1 with one of the peaks detected from the FIRST observations, sand a second one, ULX 2, possibly associated to another emission peak (see Fig. 1). Details on the peak flux and the FIRST location of the possible counterpart to ULX 1 are given in Table 1. Roberts et al. (2002) identified this source as CXOU J123029.5+413927. After the analysis of its spectral properties they suggested that the source could be a SNR. However, in a following work by Gladstone \& Roberts (2009), it was found that both flux and spectrum for the X-ray source changed, arguing against the previously suggested origin, and indicating that could be an X-ray binary instead.

\subsection{ULX 2 in NGC 5194}

NGC 5194 is an almost face-on grand-design spiral galaxy with bisymmetric spiral arms of molecular gas. It is a lowluminosity starburst galaxy with faint non-thermal radio core. It is also known as the Whirlpool Nebula and it forms an interacting system with its companion NCG 5195 (Keel et al. 1985).

The radio emission of this galaxy is not concentrated in the nucleus as in the previous case but it is extended over the galaxy disk. Sw04 listed a total of 7 potential ULXs for this galaxy. We found an evident coincidence of ULX 6 with the 


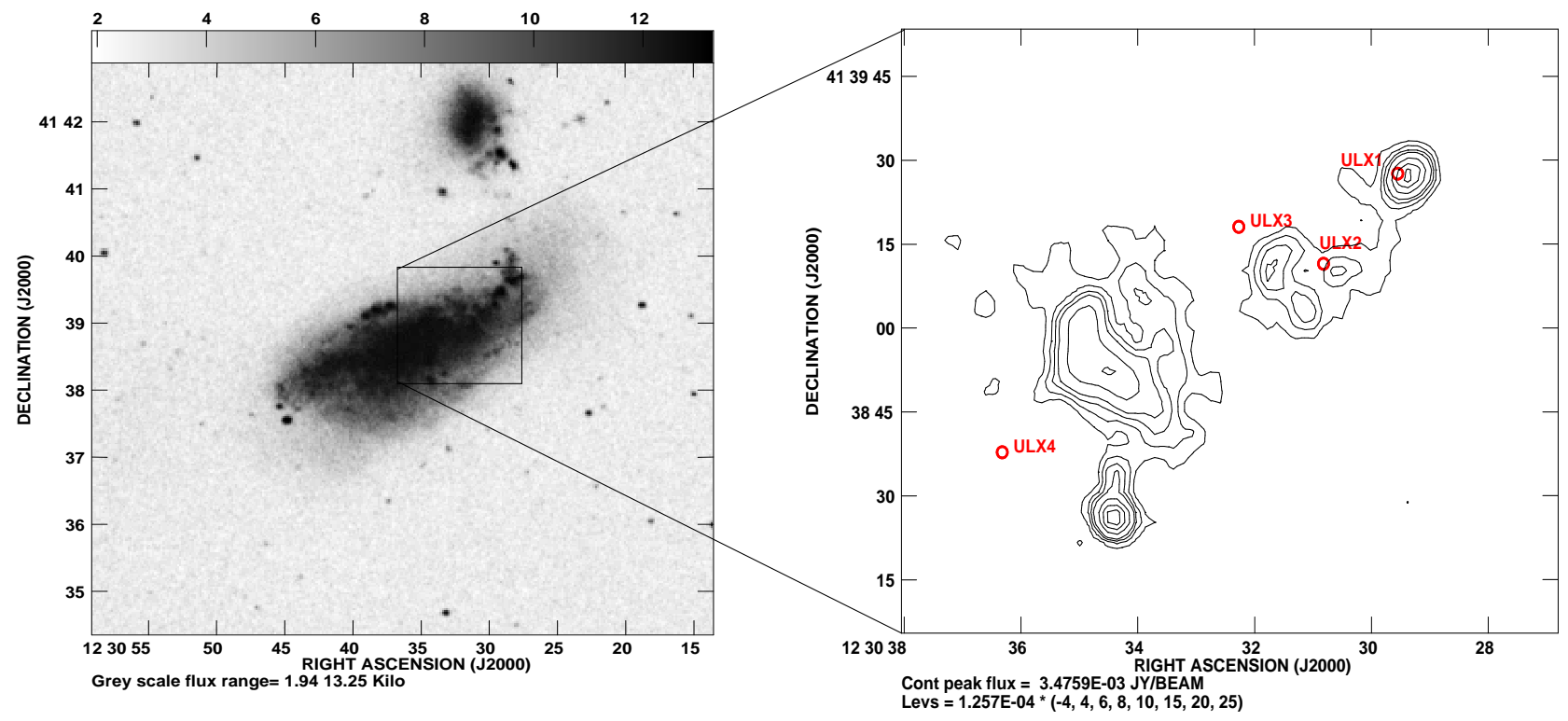

Fig. 1 Left panel: Optical DSS image for NGC 4490. Right panel: FIRST radio image contoured in units of the rms noise ( $\sim 0.15 \mathrm{mJy} / \mathrm{beam})$. These values also apply to the rest of figures. The locations of all ULX candidates listed in the Sw04 catalogue for this galaxy are shown. ULX 1 is located within the disk of NGC 4490.

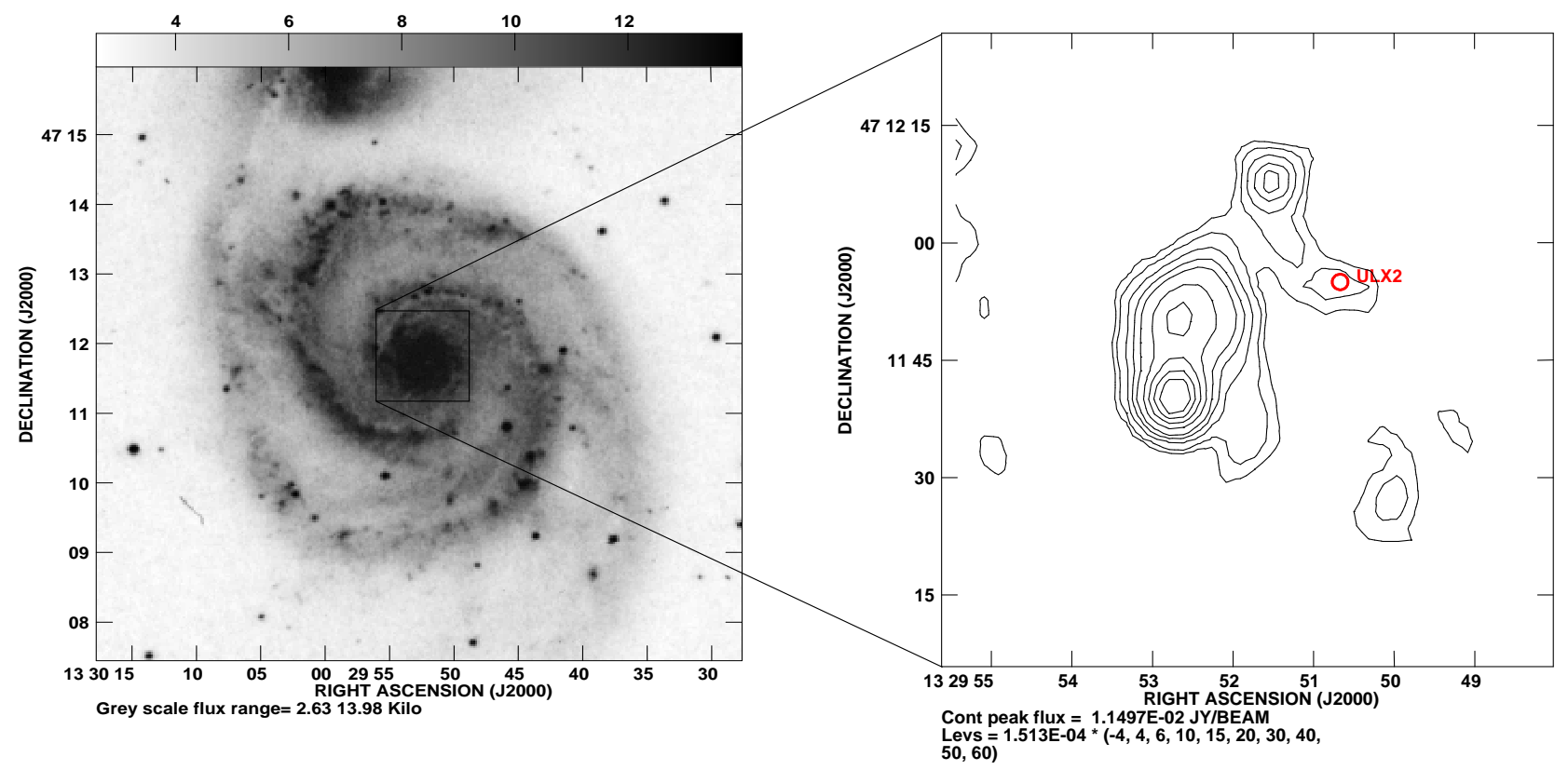

Fig. 2 As in Fig 1, now for NGC 5194. ULX 2 is located within the disk of NGC 5194.

nucleus of the companion galaxy NGC 5195. For the rest of ULXs, except for ULX 2, there was no correspondence at all between the FIRST contours and the locations of the Chandra sources. In the case of ULX 2, it is located almost coincident with a peak of radio emission, with a separation of 0.4" (see Figure 2). Details of the peak flux estimations are given in Table 1 .
This galaxy was also studied in the work by SánchezSutil et al. (2006). Three matches were found after crossidentification, but they were discarded as possible nuclear missidentifications. However, in the present work, we found that the position of ULX 2 from the FIRST contour map cannot be considered a nuclear coincidence. 


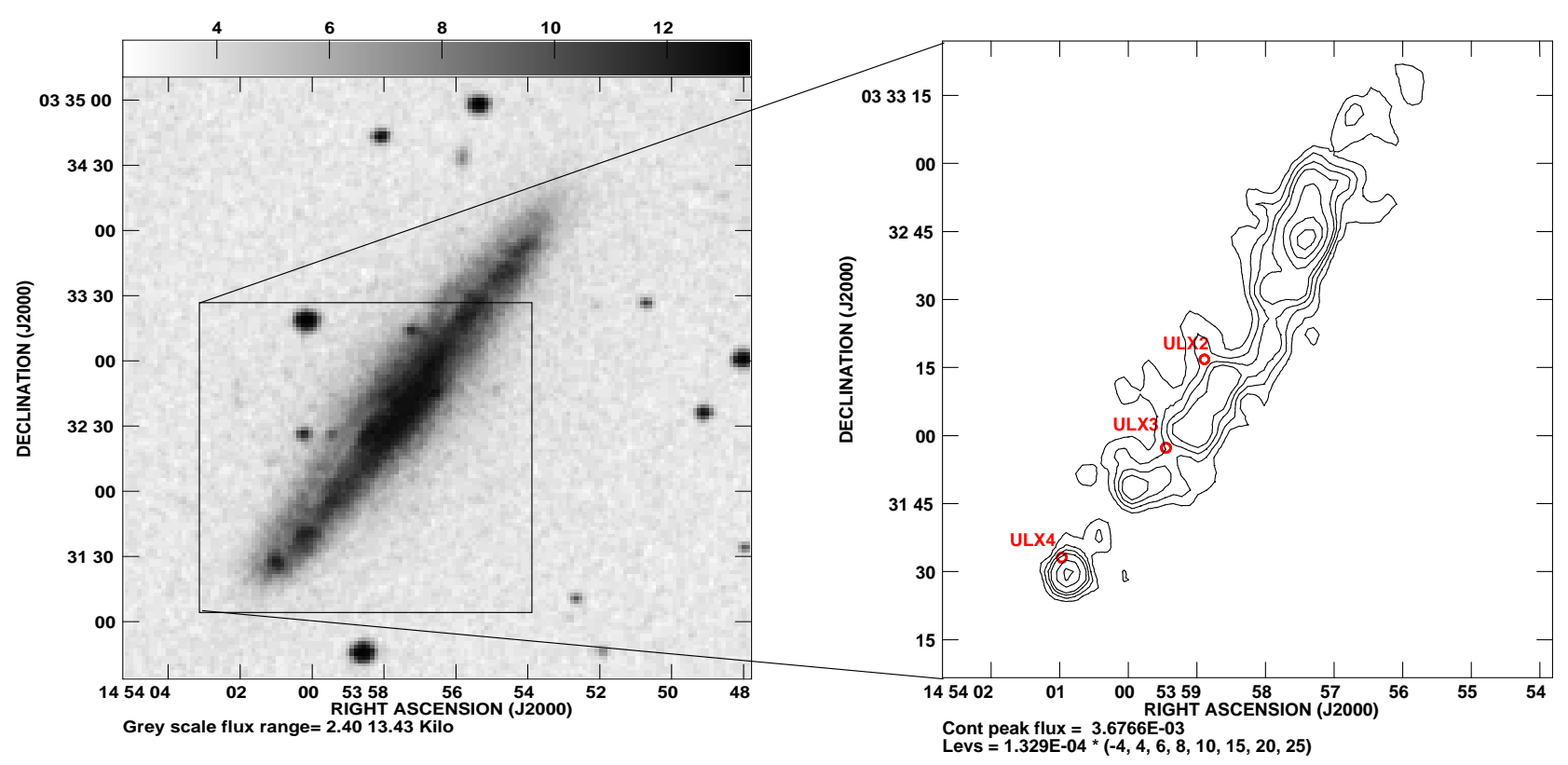

Fig. 3 As in Fig 1, now for NGC 5775. ULX 4 is located within the disk of NGC 5775.

\subsection{ULX 4 in NGC 5775}

NGC 5775 was found to be an interacting galaxy with its neighbour face-on spiral galaxy NGC 5774. Estimations based on FIR ratio by Rossa \& Dettmar (2003) suggested this galaxy to be a starburst-type galaxy.

From $\mathrm{H} \alpha$ images a possible tidal interaction has been suggested to happen, which together with the numerous compact sources at the south-east, presumably HII regions, could be an indication that star formation may be occurring well above the disk (Collins et al. 2000).

Sw04 give 4 possible ULXs associated to this galaxy, none of them coincident with the location of the nucleus given by NED. Three Chandra ULXs are located within the galactic disk in the outskirts of the contour emission map (see Figure 3). There is only one clear association of a FIRST radio emission peak for the location of ULX 4. This source is coincident with the source reported (numbered 47) in Ghosh et al. (2009). Detailed estimations for the peak and the FIRST location of the source are given in Table 1 .

\section{Conclusions}

We present results of a cross-identification of the Sw04 ULX catalogue based on Chandra data and the FIRST radio catalogue. We found FIRST radio data for 37 out of the 82 galaxies from the Sw04 sample. We ended up with 7 cases. We show in this paper three cases for which the $\mathrm{X}$-ray sources lie within 5" of the compact radio peak locations.

We did not find evidence of background QSO/AGN association based on location, as they are within the galaxy disks. None of them can be neither associated with any obvious clump of star formation visible in the optical image.
This possibility needs to be confirmed with new observational data and $\mathrm{H} \alpha$ images of the host galaxies.

Further higher-angular resolution radio observations and improved Chandra astrometry of the data are in progress. A new collection of new ULX candidates associated to more recent observed galaxies by Chandra is being compiled which will make up an extended version of the ULXs catalogue used for this work. Investigation of new cross-correlated cases will make possible to identify new cases and establish the true nature of the radio emission associated to these objects.

Acknowledgements. We thank the referee for constructive comments to improve the clarity of the paper. The research of DPR has been supported by the Universidad de Jaén (Spain). DPR acknowledges A. Castro-Tirado for useful discussions, J. Martí for suggesting the study of this set of galaxies, and finally, A. Muñoz and J.R. Sánchez for their cooperation in making the figures. This research made use of the SIMBAD database, operated at the CDS, Strasbourg, France.

\section{References}

Becker, R. H., White, R. L., \& Helfand, D. J. 1995, ApJ, 450, 559

Begelman, M., 2002, ApJ, 568, L97

Colbert, E. J. M., Mushotzky, R. F., 1999, ApJ, 519, 89

Colbert, E. J. M., Ptak, A. F., 2002, ApJS, 143, 25

Gladstone, J.C., Roberts, T.P. and Done, C., 2009, MNRAS, 297, 1836

Gladstone, J.C., and Roberts, T.P., 2009, MNRAS, 397, 124

Ghosh, K. K., Saripalli, L., Gandhi, P., Foellmi, C., Gutiérrez, C.M. and López-Corredoira, M., 2009, AJ, 137, 3263

Kaaret, P., Corbel, S., Prestwich, A. H., Zezas, A., 2003, Science, 299,365

Keel, W.C., Kennicutt, R.C., Jr., Hummel, E. et al., 1985, AJ, 90,708 
King, A.R., Davies, M.B., Ward, M.J., Fabbiano, G., Elvis, M.,2001, ApJ, 552, L109

Körding, E., Colbert, E., Falcke, H., 2005, A\&A, 436,427

Lasker, et al., 1990, AJ, 99, 2019

Liu, J.-F., Bregman, J. N., 2005, ApJS, 175, 59

Liu, Q. Z., Mirabel, I. F., 2005, A\&A, 429, 1125

Miller, N.A., Mushotzky, R.F., Neff, S.G., 2005, ApJ, 623, L109

Mushotzky, R., 2004, Prog. Theor. Phys. Supp., 155, 27

Roberts, T.P., Warwick, R.S., Ward, M.J. et al., 2002, MNRAS, 337,677

Sánchez-Sutil, J.R., Munñoz-Arjonilla, A.J., Martí, J., Garrido, J.L., Pérez-Ramírez, D., Luque-Escamilla, P., 2006, A\&A, 452,739

Swartz, D., Ghosh, K.K., Tennant, A.F., et al., 2004, ApJS, 154, 519

White, R. L., Becker, R. H., Helfand, D. J., Gregg, M. D., 1997, ApJ, 475, 479 